\title{
Arbeitsbedingungen von Kinderkrippen- Erzieherinnen in Bayern - was Erzieherinnen und Kindern Stress macht
}

\author{
Serge K. D. Sulz
}

Die in diesem Buch vielfach berichteten wissenschaftlichen Erkenntnisse zur Entwicklung von Kindern zwischen Geburt und dem Alter von drei Jahren stellen die Grundlage dieser empirischen Arbeit dar (vor allem Bindungsforschung, Säuglingsforschung, Stressforschung). Auf sie sei verwiesen, um weitere Wiederholungen zu vermeiden.

Konkrete Ausgangsbasis waren zudem einzelne mündliche Berichte von Erzieherinnen, die auf auffällig gleiche Weise mitteilten, dass die behördlichen Qualitätsanforderungen in Kinderkrippen kaum eingehalten werden (es gibt auch keine Prüf-Instanz und viele Träger von Kinderkrippen sind selbst Behörden). Zudem berichteten sie, wie die gesetzlichen Auflagen (Recht auf einen Kinderkrippenplatz quasi ab Geburt) Erzieherinnen in teilweise extreme Stress-Situationen bringen.

Darauf führten wir einige Interviews mit Erzieherinnen durch (Andrea Tichy und Serge Sulz - in diesem Buch). Diese Interviews bestätigten Art und Ausmaß der Missstände in deutschen Kinderkrippen. Die Arbeitsbedingungen schienen so, dass ein viel zu großer Anteil der Erzieherinnen ihrem Beruf nicht mehr gern nach gingen. Selbst wenn zwischen 50 und $70 \%$ der Erzieherinnen nur wenige Kritikpunkte hatten (Gehalt, zu lange Arbeitszeiten, zu viele Kinder je Erzieherin, zu geringes Alter des Kindes bei Aufnahme in die Krippe, zu kurze Eingewöhnungszeiten), dürfen wir die mit ihren Arbeitsbedingungen unglücklichen bzw. durch diese Arbeitsbedingungen gestressten Erzieherinnen (30 bis $50 \%$ ) nicht einfach übersehen. Diese erzeugen Stress, der sich auf die Kinder auswirkt, ebenso sehr wie der Stress der berufstätigen Eltern. Das sind die, die sich nicht im von der Politik gewünschten Ausmaß anpassen können. Sie kündigen, wenn sie es nicht mehr aushalten, so dass eine erhöhte Fluktuation des Personals entsteht sowie ein Fachkräftemangel, der den Stress der zurückgebliebenen zusätzlich erhöht. Wenn es gelingt, für Erzieherinnen Stressprävention anzubieten, ist ein wichtiger Schritt aus der Misere heraus getan.

Der nächste Schritt war deshalb der Versuch, repräsentativere Daten zu erhalten. Hierzu wurden Kinderkrippen in Bayern angeschrieben, deren Adressen vom Bayerischen Landesamt für Statistik stammten.

Das Anschreiben enthielt folgende Angaben: 
„Wir führen eine wissenschaftliche Untersuchung durch, die der Stressforschung dient. Wir wollen Stressquellen und Stressauswirkungen in Arbeitsstätten untersuchen und stellen dazu Fragen unter dem Vorzeichen von subjektiv empfundenen Arbeitsbedingungen in Kinderkrippen.

Ziel ist letztendlich ein Stress-Präventionsprogramm zu entwickeln, das spezifisch auf die Arbeitssituation der Kinderkrippen-Erzieherin und der Leiterin ausgerichtet ist. Deshalb interessieren auch nicht die objektiven Arbeitsbedingungen, sondern nur wie diese erlebt werden. Denn wie etwas erlebt wird, kann schon ein wichtiger Stressfaktor sein.

Wir wollen uns auf die Erziehung in Kinderkrippen beschränken, in denen nur Kinder bis drei Jahre betreut werden, also nicht auf altersgemischte Abteilungen/Gruppen und auch nicht auf Kindergärten.

Wenn es gelingt, das herauszufiltern, was Dauerstress und die daraus resultierenden Erkrankungen und Kündigungen verursacht, können wir sehr spezifisches Stress-Management entwickeln und Ihnen zugutekommen lassen.

Wir würden uns sehr freuen, wenn Sie und Ihre Mitarbeiterinnen einzeln und getrennt die Fragen online beantworten würden. Dazu müssen Sie auf die sozialwissenschaftliche Forschungs-Webseite gehen: https://www.soscisurvey.de/Kita-Erzieherin."

In der Einleitung zum Fragebogen wurde noch einmal auf mögliche ungünstige Bedingungen hingewiesen: „Im Fokus der Diskussion um die Qualität von Kitas stehen die Kinder und ihre Mütter. Nach den ErzieherInnen fragt niemand. Es kann sogar sein, dass ihnen die Schuld an der Misere zugeschoben wird: „Wenn Ihr bessere Arbeit machen würdet ..." oder „Warum können Sie das nicht noch zusätzlich machen?“. Während der (Alters-) Pflegenotstand in Presse und Bevölkerung inzwischen angekommen ist, mag sich niemand um die Arbeitsbedingungen der ErzieherInnen kümmern."

Die für Kinderkrippen zuständige Behörde der Stadt München (als Bayerns größter Betreiber von Kinderkrippen) hat sich geweigert, die Fragen an ihre Erzieherinnen weiterzugeben - mit der Begründung, dass diese beruflich so belastet seien, dass ihnen der Zeitaufwand für den Fragebogen nicht zugemutet werden könne. Wohlgemerkt, ein Fragebogen, der helfen soll, den Stress der Erzieherinnen zu reduzieren.

\section{DIE BEFRAGUNG}

Es haben 205 Erzieherinnen, die in Kinderkrippen mit Kindern unter 3 Jahren arbeiten, den Fragebogen vollständig ausgefüllt. Wer im Kindergarten arbeitet oder mit altersgemischten Gruppen wurde nicht mit einbezogen. Der vollständige Fragebogen mit allen genauen Häufigkeiten je Antwort-Alternative befindet sich im Anhang dieses Buches. Hier werden nur die für das Verständnis der Situation der Erzieherinnen wichtigen Variablen aufgegriffen und diskutiert.

$99 \%$ der Erzieher waren weiblich. Die Altersverteilung war wie folgt: 


$\begin{array}{ll}\text { Alter Erzieherin } & \text { Prozent } \\ 20 \text { bis } 25 & 21 \% \\ 26 \text { bis } 30 & 10 \% \\ 31 \text { bis } 35 & 13 \% \\ 36 \text { bis } 45 & 20 \% \\ 46 \text { bis } 55 & 17 \% \\ 56 \text { bis } 65 & 19 \%\end{array}$

Der überwiegende Teil war über drei Jahre lang in Kinderkrippen tätig. Die Hälfte der Befragten waren Leiterinnen der Kinderkrippe. Dies lag daran, dass die Leiterinnen angeschrieben wurden und viele von Ihnen den Fragebogen selbst ausgefüllt haben. Dadurch geht natürlich die Leitungsperspektive mit deren spezifischen Werten und Anliegen sehr stark in das Ergebnis ein. Meistens handelte es sich um Träger, die nur eine oder wenige Kinderkrippen unterhielten. Die Hälfte der Träger war gemeinnützig. Und fast die Hälfte der für eine Kinderkrippe zuständigen Vorgesetzten der Kinderkrippenleiterin hatte einen sozialen Beruf, war also prinzipiell nicht primär kaufmännisch oder verwaltungstechnisch ausgerichtet.

\section{DIE KINDERKRIPPEN-LEITERIN}

Sie hat mehrheitlich mehr als drei Jahre Kinderkrippenerfahrung mit mehr als drei Jahren Leitungserfahrung. Die Frage nach ihrem Stresslevel haben leider nur 171 Befragte beantwortet. Zweidrittel gaben an, dass es viel zu viel Stress sei bzw. der Stress auf Dauer so nicht auszuhalten sei. Das Qualifikationsprofil wies gute Werte bezüglich Organisation, Umgang mit Personal, Eltern und Kindern auf. Aber der fürsorgliche Umgang mit sich selbst wurde negativ bewertet. Bei der Hälfte der Leiterinnen ist es so, dass die Arbeitsbedingungen es ihnen nicht erlauben, gut für sich zu sorgen (z. B. Burnout-Prophylaxe). Dazu fehlt der Leiterin an erster Stelle Wertschätzung für sie und ihre Leistung und dass ihr Stress abgenommen wird. Sie hätte auch sehr gern mehr Zeit für die Kinder. Ihre größte Angst ist, dass sie nicht genügend Erzieherinnen bekommt, dass das Teamklima unter dem Stress leidet und dass sie ihre Arbeit nicht mehr schafft.

\section{DIE KINDER IN DER KINDERKRIPPE}

Die Kinderkrippen der befragten Erzieherinnen sind zu einem Drittel klein (bis 20 Kinder), mittelgroß (bis 30 Kinder) und groß (31 bis 50 oder mehr Kinder). Nur ein Drittel der Kinderkrippen haben keine Kinder unter einem Jahr. Kinder zwischen einem und zwei Jahren waren sehr häufig in der Krippe, ebenso viele wie zwischen zwei und drei Jahren. Die Dauer der täglichen Unterbringung von Kindern zwischen null und einem Jahr war überwiegend 8 oder mehr Stunden (sie blieben ebenso lang in der Krippe wie die ein Jahr älteren Kinder): 
Wie lange bleiben Kinder, die noch kein Jahr alt sind, pro Tag?

$\begin{array}{ll}\text { Zahl Kinder } & \text { Dauer des tägl. Aufenthalts } \\ \mathbf{1 7} & \text { bis zu } 4 \text { Stunden pro Tag } \\ \mathbf{3 5} & \text { bis zu 6 Stunden pro Tag } \\ \mathbf{7 4} & \text { bis zu 8 Stunden pro Tag } \\ \mathbf{2 3} & \text { bis zu 10 Stunden pro Tag } \\ \mathbf{1} & \text { länger als 10 Stunden pro Tag }\end{array}$

Dabei ist zu berücksichtigen, dass (wohl aus organisatorischen und finanziellen Gesichtspunkten) 32 Kinderkrippen von den Eltern eine Ganztagsunterbringung forderten und eine Halbtagsunterbringung ablehnten.

Während einer Schicht war eine Erzieherin überwiegend für vier bis acht Kinder zuständig. Nur dreizehnmal wurde berichtet, dass sich nur drei Kinder eine Erzieherin teilen mussten. Engpässe, in denen zu wenig Erzieherinnen da waren, dauerten bei der Hälfte der Krippen vier Wochen oder länger. Das ist mit das alarmierendste Ergebnis dieser Studie. Für ein kleines Kind ist so ein Mangel nicht verkraftbar. Während so einem Engpass wurde 15-mal berichtet, dass bis zu zehn Kinder auf eine Bezugsperson kamen (wochenlang!) und immer noch 26-mal bis zu 15 Kinder. Übereinstimmend mit der Beobachtungsstudie von Samel und Wedlich (in diesem Buch) erhielten die meisten Kinder nicht mehr als 30 Minuten tägliche persönliche Zuwendung. Auch das ist alarmierend!

\section{DIE KINDERKRIPPEN-ERZIEHERINNEN}

Es wird über wenig Personalwechsel berichtet (falls die Frage richtig verstanden wurde - es wurde gefragt, wie lange sie schon in der Kinderkrippe arbeiten und nicht wie lange schon in dieser Kinderkrippe). Der Urlaub wird zu $90 \%$ außerhalb der Ferien genommen, weshalb fast alle Kinder durch Bezugspersonenwechsel gestresst werden. Ein Viertel der Kinderkrippen hat keine Betriebsferien, d. h. Eltern müssen nicht wegen ihren Kindern Urlaub nehmen.

Mehr als dreiviertel der Erzieherinnen berichten über zu viel Stress, mit dessen Bewältigung sie allein gelassen werden. Zugleich geben sie an, dass sie nicht über genügend Stressbewältigungsstrategien verfügen und deshalb nicht ausreichend Selbstfürsorge betreiben können. Sie sind ganz und gar damit beschäftigt, dafür zu sorgen, dass es ihren Kindern gut geht und diese sich wohl fühlen. Sie würden sich sehr gern dem einzelnen Kind mehr zuwenden, wenn es ihre Arbeitsbelastung zulassen würde. Aber auch die gute Zusammenarbeit im Team ist ihnen sehr wichtig. Sie würden sich zu all dem in der Lage sehen, wenn es ihnen ermöglicht werden würde. Stressreduzierend wäre für sie das, was sie am dringendsten brauchen: ein besserer Personalschlüssel und angemessenes Gehalt. Aber ebenso sehr würde ihren Stress 
reduzieren, wenn nicht so eine große Verantwortung auf den Schultern der Einzelnen lasten würde. Sie müsste dann nicht so viel Angst haben, dass einem Kind etwas passiert. Und auch dass Teamzusammenhalt vor lauter Stress immer schlechter wird. Sie wünschen sich zudem eine wirksamere Befähigung ihren Berufsstress zu bewältigen. Zum Beispiel dadurch, dass ein Personalschlüssel von 1 zu 3 wirklich und auch durchgängig eingehalten wird. Bezüglich des Umgangs mit dem Stress der Kinder sehen sie sich in der Lage, diesen zu erkennen und zu reduzieren.

Die überwiegende Mehrheit ist dafür, dass die Einrichtung sechs Wochen im Jahr schließt, damit die Kinder in dieser Zeit bei ihren Eltern sein können. Allerdings wollen sie nicht, dass außerhalb dieser Schließzeit Urlaubsverbot herrscht. Sie wollen individuell Urlaub machen können.

Die Befragten sind eindeutig bildungs- und erziehungsorientiert. Sie glauben, dass von der Einrichtung an Kinder unter 3 Jahren herangetragene Bildungsmaßnahmen bedeutsam sind. Auch wenn in einer Frage angedeutet wird, dass das kindliche Gehirn vor dem Alter von 4 Jahren noch über wenig kognitive Fähigkeiten verfügt. Eine gegenteilige Auffassung und Vorgehensweise wäre, dem Kind freie Exploration seiner kleinen Welt zu ermöglichen, so dass Lernen aus seiner Neugier heraus erfolgt. Aber die Differenz kann auch daran liegen, dass vielleicht gar keine straffen Lernprogramme angesetzt werden sollen.

\section{DIE ELTERN}

Bei Eltern hält sich das Anstrengende und Unterstützende die Waage. Den Eltern werden Empfehlungen gegeben, sobald deutlich wird, dass sich ihr Kind in einem Dauerstress befindet. Oft bleibt nur wenig Zeit sich mit den Eltern auszutauschen. Andererseits findet sich doch immer wieder Zeit und auch Interesse der Eltern. Bei dem was Eltern berichten wollen, fehlt ebenfalls teils die Zeit. Wissen der Eltern über die Bedeutung der Bindungssicherheit ist teilweise vorhanden, teilweise aber recht gering. Ebenso können sie die Bindungssicherheit ihres Kindes nur zum Teil gut einschätzen. Dabei scheinen einige Kinder diesbezüglich recht stabil zu sein.

Nach diesen Multiple-Choice-Fragen wurde noch nach weiteren wichtigen Punkten gefragt: Was noch wichtig ist und im Fragebogen nicht oder nicht ausreichend angesprochen wurde.

Nachfolgend werden die freien stichwortartigen Nennungen aller Erzieherinnen wiedergegeben, weshalb Wiederholungen auftreten und die Liste nicht nach Themen geordnet ist. 


\section{DAS WICHTIGSTE}

- Ich finde es wichtig die Gruppen zu verkleinern. Mehr Erzieher pro Gruppe sind nicht hilfreich, da dann zu viele Bezugspersonen in einem Raum sind. Es wäre wirklich besser höchstens 15 Kinder im Alter von drei bis sechs und acht bis höchstens zehn Kinder in einer Gruppe unter drei zu betreuen. Der Lärmpegel ist alleine schon anstrengend genug für die Kinder.

- Ständige Krankheitsausfälle der Erzieher.

- Alter und Länge der Anwesenheit: Kinder sind länger als Bezugserzieher in der Krippe.

- Eingewöhnungsmodelle.

- Inklusion.

- Fachkräftemangel: Wieviel offene Erzieher Stellen haben sie zur Zeit? Meine Antwort: 2 Stellen sind über 6 - 8 Monate nicht besetzt, da keine Bewerbungen eingehen.

- Finanzielle Mittel zur altersgerechten Ausstattung der Räume.

- Kinder kommen immer kränker in die Kita und die Eltern nehmen Krankheiten nicht ernst. Kinderärzte schätzen Krankheiten ebenfalls nur noch lapidar ein und erlauben Kindern den Kitabesuch.

- Eignung des offenen Konzepts.

- Mangelnde Ausbildung insbesondere in der Kinderpflege wirkt sich negativ auf die pädagogische Arbeit aus, kostet viel Energie.

- Finanzielle Unterstützung der Politik.

- Krankheitsausfälle und deshalb Personalmangel trotz gutem Schlüssel.

- Weiterbildung in verschiedenen Themenbereichen von Team und Leitung.

- Personalengpässe.

- Die Eltern sehen oft nicht wie wichtig ihre eigene Stärke für die Entwicklung ihres Kindes ist.

- Mehr Zeit für die Eingewöhnungszeit.

- Die Ausstattung der Krippe schwächt Stress oder fördert ihn.

- Die räumliche Aufteilung und Ausstattung müsste ein großes Thema sein. Viele Krippen sind in ehemaligen Kindergartenräumen untergebracht und daher ungeeignet in der täglichen Arbeit (lange Wege ins Bad, ein Schlafraum der zwischen zwei Gruppenräumen liegt usw.).

- Päd. Fachkräfte übernehmen noch zusätzlich die komplette Hauswirtschaft; dies sollte nicht noch eine zusätzliche Belastung sein (Wäsche waschen und aufhängen, Tische und Böden nach den Mahlzeiten putzen, Geschirr nach den Mahlzeiten säubern, sensible Bereiche desinfizieren usw.).

- Qualifizierung/Ausbildung Krippenpersonal.

- Wir sind in einer Mutter-Kind-Abteilung im geschlossenen Vollzug (JVA) tätig. Daher sind einige Fragen nicht zutreffend.

- Der Faktor eines einjährigen Kindes sollte nicht gleichgestellt sein, mit dem eines 2,5 Jahre alten Kindes.

- Lautstärke und psychische Belastung.

- Zu wenig Zeit der Eltern für Eingewöhnung.

- Springer Kräfte in den Einrichtungen. 
- Viel zu wenig Gehalt.

- Der Zeitfaktor einer Eingewöhnung. Oft haben/wollen/können die Eltern diese Zeit für eine gute Eingewöhnung nicht aufbringen.

- Entwicklungsgespräche.

- Personalschlüssel mit FACHPERSONAL aufstocken.

- Welche Stellung haben die Kinderpflegerinnen? Hier wird immer nur von Erzieherinnen gesprochen. Wir haben aber nur 2 Erzieherinnen und 5 Kinderpflegerinnen, sowie eine SPS Praktikantin in der Krippe.

- Qualität der Ausbildung und Persönlichkeiten der Pädagogen.

- Wie kann man dem Stresspegel entgegensteuern.

- Schließtage. Außerhalb der Schließtage werden bei uns Kinder zwei Wochen in den Urlaub geschickt, welchen die Eltern bestimmen. Die Frage mit Ferienzeit ist für den Befragten eher verwirrend.

- Verpflichtende gesetzliche Richtlinien für Kleinkindgruppen (Alter, Größe Betreuungsschlüssel).

- Wie viele Angebote werden an einem Tag durchgeführt? D. h. haben die Kinder die Möglichkeit zu wählen, ob sie turnen, musizieren, kreativ gestalten, kochen und backen, schwimmen, usw. machen möchten und mit wem (Kindern, wie Erziehern)?

- Wie gehe ich mit Stress um, welche Strategien helfen im Alltag?

- Vor- und Nachbereitungszeit für Erzieherinnen.

- Die räumlichen Bedingungen.

- Das man ganz schlecht eine unbefristete Vollzeitstelle findet. Nach meiner Ausbildung habe ich nur Jahresverträge oder Vertretungsstunden. Obwohl Erzieherinnen anscheinend fehlen, werden keine eingestellt.

- Gesellschaftliche Akzeptanz.

- Gute Ausbildung des päd. Personals.

- Kranke Kinder, die in die Einrichtung gebracht werden.

- Einhaltung der vorgegebenen Qualitätsmerkmale bezogen auf die Anzahl der Kinder pro Gruppe sowie des Personalschlüssels.

- Stress bei Kindern aufgrund von falschem oder zu viel Fernseh- Handy(spiele)-konsum!

- Die Ausbildung muss verbessert werden. „Die Besten für die Kleinsten” und damit auch die Bezahlung.

- Krippenkinder erleben nicht nur manchmal einen Wechsel der Bezugserzieher, sondern sie sind im Frühdienst mit einer anderen Erzieherin und anderen Kindern zusammen als im Spätdienst.

- Mittagsschlaf in der Krippe - Cortisolspiegel des Kindes.

- Anleitung von Praktikanten.

- Achtsamer Umgang mit den Kindern.

- Gruppenstärke: 12 Kinder in einer Gruppe sind zu viel, daraus folgt z. B. hohe Lautstärke, wenig Bewegungsraum für Kinder ...

- Die verschiedenen Ausbildungen der Mitarbeiter.

- Wenn kranke Kinder die Einrichtung besuchen

- Die Gestaltung der Räume - Reizüberflutung ...

- Oft schätzen die Eltern unsere Arbeit zu wenig wert. 
- Die Ausbildung von Krippenpädagoginnen ist teilweise. sehr mangelhaft, das sollte (muss) verbessert werden.

- Kleinere Gruppen und weniger Bezugspersonen.

- Erholungsphasen für Erzieherinnen.

- Die Eingewöhnung ist zentral für die gute Bindung. Ist die Eingewöhnung gut verlaufen und hat das Kind währenddessen die weiteren Erzieher der Gruppe gut kennengelernt, wird jeder zur Bezugsperson und somit sind Urlaube und Krankheiten für die Kinder kein Drama.

- Berufstätigkeit der Mütter ist gesellschaftlich und politisch erwünscht.

- Es ist die Dauer, die ein Kind individuell braucht (mit Eltern, mit Kindern, anderen Bezugspersonen).

- Möglichkeiten der Stressreduzierung durch optimale, harmonische Raumgestaltung.

- Kinder unter 1,5 Jahren sollten anders im Personalschlüssel berücksichtigt werden als ältere Kinder (Faktor mind. 4,0).

- Personalschlüssel ist zu niedrig, Hauswirtschaftsstunden zu gering, Betten beziehen, Vesper richten etc. benötigt viel Zeit.

- Gruppengröße, Hilfspersonal.

- Bezahlung der Erzieher.

- Erzieherpraktikanten werden als volles Teammitglied gerechnet, brauchen aber noch selber viel Unterstützung und sind viele Tage nicht in der Einrichtung.

- Unterstützt die Leitung ihr Personal in den Gruppen und hilft dort im Notfall mit?

- Leitung in Doppelfunktion - Kinder und Leitung.

- Die Öffnungszeiten sollten begrenzt sein - von 24 Stunden Öffnung halte ich nichts.

- Bei Personalmangel darf man keine Überstunden aufbauen, sondern legt die einzelnen Gruppen zusammen, so dass es kaum noch zu tragen ist ... Warum?

- Besondere Situation der Eingewöhnung.

- Genug Eltern-Kindurlaube.

- Zur Stressreduzierung der Kinder und des Personals wäre es sinnvoll den Gewichtungsfaktor für Krippenkinder zu splitten. Kinder von 0 bis 3 Jahren haben bis jetzt alle einen Gewichtungsfaktor von 2,0. Optimaler wäre bei 0 - 1jährigen ein Faktor von 4, bei 1 - 2jährigen ein Faktor von 3 und bei 2 - 3jährigen ein Faktor von 2. Das wäre ein sehr wichtiger Schritt in die richtige Richtung.

- Eingewöhnung. Wie verläuft sie und wie lang ...

- Mehr Personal in den einzelnen Gruppen, dass mehr Zeit für das Kind und am Kind ist und wenn mal Engpass ansteht, dass es nicht so ins Gewicht fällt.

- Eingewöhnungszeiten im September und Oktober ist sehr anstrengend, besser über das Jahr verteilen.

- Qualifizierung und Eignung des Personals.

- Die räumlichen Bedingungen (ob es genügend und ausreichend Platz für z. B. Rückzug, Bewegung, Schlafen usw. gibt).

- Beobachtung und Dokumentation, mehr Zeit dafür. 


\section{DAS ZWEITWICHTIGSTE}

\section{Ein zweites wichtiges Thema ist noch:}

- Die bessere Ausbildung von Erziehern. Viele sind nicht wirklich für den U3 Bereich ausgebildet und denken sie müssen pädagogische Angebote machen. Ich bin immer wieder erstaunt, wie wenig sie über Entwicklungspsychologie, Bindung und den Umgang mit kleinen Kindern wissen.

- Kranke Kinder in der Kita.

- Eingewöhnungszeit.

- Kinder unter 3 Jahren werden immer häufiger über 8 h in der Kita abgeschoben. Manchmal kommen dann zusätzlich auch noch Tagesmütter.

- Gestaltung der Eingewöhnung.

- Inklusion.

- Gesundheitspräventionsangebote gestellt durch Träger.

- Wertschätzung der Arbeit durch die Eltern.

- Helicopter-Eltern.

- Bezuschusste Kleidung würde für Erleichterung sorgen: Knie sind schnell durchgewetzt durch die viele Arbeit am Boden, in der Lohnsteuer wird das leider nicht geltend gemacht.

- Erzieher und Kinderpfleger sollten alle eine berufliche Qualifikation für Krippenarbeit abschließen. Dafür müssten finazielle Mittel zur Verfügung gestellt werden.

- Immer mehr Kinder mit stark wahrnehmbaren Auffälligkeiten (Sprachentwicklung, extremes Beißen, Regel- und Distanzlosigkeit) besuchen unsere Regeleinrichtungen; entgegen der öffentlichen Meinung erfährt man hier kaum und nur wenig hilfreiche Unterstützung; die Eltern verweigern oft die Zusammenarbeit und geben uns die Rückmeldung, wir wären das Problem.

- Mehr zu Erzieherinnen/Kinderpflegerinnen als Fragen über die Leitung da diese meist nicht nur Kinderkrippen leiten.

- Kranke Kinder in der Einrichtung.

- Freistellung der Leitungen vom Gruppendienst.

- Ständige Überstunden.

- Überstunden und Mehrarbeit für die Erzieherinnen ist kein Hobby!

- Dauer der Vorbereitungszeit.

- Konzept der Kita sollte praktisch umsetzbar sein - nicht nur theoretisch gut klingen! (Individuell können wir in der Krippe ganz oft nicht leisten!).

- Kinder dürfen laut Gesetz nicht länger als 10 Stunden am Tag betreut werden.

- Weiterbildungen, Teamtage.

- Wie groß, bzw. klein ist diese Kleingruppe bei dem jeweiligen Angebot, z. B. ein Kind zu einer Erzieherin, oder zwei Kinder, usw.

- Wie Fülle ich meinen Akku auf.

- Leiterin sollte mehr Interesse für die Praxis zeigen.

- Die verlängerten Öffnungszeiten. 
- Dass jede/r Erzieher/in, die/der mit Kindern unter drei Jahren arbeitet, eine Zusatzqualifikation U3 hat und sich kontinuierlich fortbilden kann.

- Fehlende Tagesstruktur (als Orientierungshilfe) bei Kleinstkindern.

- Zusätzliches Fachpersonal (Psychologinnen, Therapeutinnen, etc.) müssen das Kita-Team direkt unterstützen in multidisziplinären Teams.

- Manche vorgegebene Antworten spiegelten leider nicht den tatsächlichen Zustand wieder.

- Mittagsschlaf der Kinder, Eltern wollen nicht, dass die kleinen Kinder schlafen gibt Stress.

- Dass Eltern oft nicht die Wahrheit sagen z. B. was hat das Kind?

- Bewegungsmöglichkeiten für die Kinder und Mitarbeiterinnen.

- Die Elternarbeit kommt im Zusammenhang mit Aufklärung der Eltern über Bildung und Familie doch zu kurz.

- Eltern, die uns als Fachkräfte ernst nehmen und respektieren.

- München und Umgebung ist sehr teuer geworden, Mieten und Immobilienpreise steigen.

- Möglichkeit der Gruppenverteilung durch vorhandene Räumlichkeiten, neben dem Gruppenraum.

- Die Leitung sollte ab einer bestimmten Gruppenzahl nicht mehr im Schlüssel dabei sein (ab 2 - 3 Gruppen mindestens halbtags, ab 4 Gruppen komplett freigestellt).

- Gehen alle Kinder jeden Tag an die frische Luft? Und wie lang?

- Räumlichkeiten.

- Bildungsdokumentation.

- Wie ist die rechtliche Lage bei Personalmangel (z. B. bei Krankheitswellen) - wie viele Kinder dürfen/müssen Erzieher dann betreuen?

- Behinderte oder von Behinderung bedrohte Kinder werden in der Personalberechnung nicht berücksichtigt.

- Die Fachkräfte brauchen ausreichend Zeit um Dinge vorzubereiten und zu planen.

- Überstunden abbauen laut Anweisung der Leitung: Eine Frechheit. Warum darf sie bestimmen wann wir Mitarbeiter frei haben oder früher/später gehen müssen.

- Ausbildung von Praktikanten, Einarbeiten von Krankheitsvertretung.

- Zeitweise Freistellung der Leitung, damit alles im Büro erledigt werden kann und dadurch niemand in der Gruppe fehlt.

- Die Kinder werden oft krank in die Krippe gebracht.

- Vorgabe bei der Einrichtung einer Krippe.

- Die Qualifikation der Mitarbeiter in der Krippe (viele sind z. T. noch in der Ausbildung und haben somit keine Vorerfahrungen).

- Elternarbeit.

- 


\section{DAS DRITTWICHTIGSTE}

\section{Ein drittes wichtiges Thema ist noch:}

- Kollegialer Austausch, Zeit für Vorbereitung, Zeit für Entwicklung von Konzepten usw.

- Überkritische Eltern.

- Personalschlüssel 1 zu 3.

- Wertschätzung der Arbeit durch den Träger/bessere Bezahlung.

- Der Beruf des Erziehers in der Krippe müsste gesellschaftlich höher gestellt werden. Hier werden entscheidende Grundlagen für die Entwicklung der Kinder gelegt.

- In den Medien wird das Thema Bildungseinrichtungen komplett falsch dargestellt; es sollte hier einmal ein Bericht erscheinen, der auf Berichten der Erzieherínnen beruht und nicht auf medienwirksamer Publicity.

- Körperliche Belastung, kleine Stühle, viel heben und tragen der unter 1 Jährigen sowie ältere und schwerere Kinder.

- Immer höhere Ansprüche seitens der Eltern an das Personal.

- Personalschlüssel? Das kann man nicht über eine Frage klären. Wir sind in einem Einfamilienhaus mit einer Gruppe (16 Kinder). Hinzu kommen 3 Erzieher und 2 Kinderpfleger sowie Reinigungspersonal + Hausmeister...

- Mindestens 25 Schließtage.

- Führt immer die gleiche Erzieherin, für das gleiche Kind, die gleiche Art des Angebotes durch? Z. B. eine Erzieherin ist immer für die Bewegungsbaustelle zuständig.

- Welche Bedingungen müssen geändert werden, um entspannter zu arbeiten.

- Es werden immer mehr Dinge verlangt (Dokumentation, Portfolio, Entwicklungsbögen etc.) aber es gibt keine bezahlte Stundenerhöhung. Das läuft alles nebenher und man arbeitet sogar in der Freizeit, weil man es ansonsten gar nicht schafft.

- Wissen um die Qualitätsvoraussetzungen bei Kindern U3 und der Konsequenzen, wenn diese nicht vorhanden sind. Aber die sind ja „so süß!” und noch zu klein, um zu lernen (was auch immer darunter verstanden wird).

- Mangelnde Flexibilität um auf die vorrangigen Bedürfnisse der Kinder eingehen zu können.

- Kinder müssen länger krank bleiben und gesunden dürfen.

- Hier ist die Rede von reiner „Krippe”, jedoch sind die Krippen zumeist in große Kitas integriert, die Erzieher wechseln auch zwischen Kindergarten und Krippe, und zu manchen Zeiten sind die größeren Kinder mit im Krippenbereich zu beaufsichtigen (z. B. Früh- und Spätdienst).

- Unzuverlässige Kolleginnen.

- Die Haltung der Pädagogen zur Betreuung von Kindern unter 3 Jahren.

- Die Finanzierung über die Eltern ist tlw. nicht möglich, weshalb Kindern der Zugang zu Bildungseinrichtungen verwehrt bleibt. 
- Wiedereinstieg in den Beruf der Mütter muss/müsste gesichert werden.

- Sicheres Kontingent an Vorbereitungszeit zur optimalen Gestaltung der Bildungsarbeit.

- Eltern denken mittlerweile, dass Ganztagesbetreuung besser für die Kinder ist als die Betreuung zu Hause.

- Ernährung.

- Zügige Stellenbesetzung.

- Das Wohlbefinden der Kinder sollte in jedem Fall an erster Stelle stehen.

- Warum darf eine Leitung Überstunden aufbauen, wann und wie viel sie will? Ihre Stunden rumschieben, wie es ihr gerade passt. Und Mitarbeiter dürfen das nicht. Und noch dazu über $10 \mathrm{Std}$. Arbeit am Tag und nur eine halbe Stunde Pause machen.

- Hausinterner Springer wäre vom Vorteil, damit Engpässe abgedeckt sind.

- Weiterbildungen für Krippenkinder.

Ende des freien Teils der Erzieherinnen-Befragung

Die Erzieherinnen haben den freien Teil der Befragung reichlich genutzt, um problematische Themen anzusprechen, die im ersten Teil des Fragebogens nicht ausreichend belichtet wurden. Jedes dieser nur stichwortartig genannten Themen könnte und müsste noch vertieft werden, um die Gesamtsituation bezüglich der Arbeitsbedingungen der Erzieherinnen in Kinderkrippen zu erfassen.

Ein Fragebogen in einer notwendigen Nachfolgestudie müsste diese Themen einschließen. Sie spiegeln noch viel deutlicher die Arbeitsbelastungen der Erzieherinnen wieder als durch Multiple Choice-Fragen erfasst werden kann.

Wichtig ist, dass mit dieser Befragung nicht die objektiven Umstände in den Kinderkrippen erfasst wurden. Es wurde - wegen der Zielrichtung „subjektiv erlebter Stress" nur zur Sprache gebracht, wie Erzieherinnen ihre Arbeit erleben.

Die in diesem Buch vorgestellte Kinderkrippen-Ampel weist auf zahlreiche Möglichkeiten hin, die helfen, die Arbeitsbedingungen von Krippen-Erzieherinnen in dem Ausmaß zu verbessern, wie es notwendig erscheint. Sie kann als Leitlinie gelten, die die Anliegen und das Recht auf einen stressärmeren Beruf der Erzieherin in einer Kinderkrippe gut abbildet. Die Ampel zeigt Lösungswege aus der Krippenmisere auf und die Verwirklichung ihrer Empfehlungen wäre der sicherste Weg zur Stressreduktion von Erzieherin, Kind und Eltern. Sie kann ebenso gut als Audit-Fragenliste für das Qualitätsmanagement eingesetzt werden (Tabelle 1).

Es wurde sehr deutlich, dass viele Erzieher in Kinderkrippen keine Erfahrung und vor allem keine Zusatzausbildung für die Betreuung von U3-Kindern (Kinder unter 3 Jahren) haben. Eine Zusatzausbildung (berufsbegleitend im Umfang von z. B. 180 Stunden inklusive Selbsterfahrung) ist neben Supervision und der Umsetzung obiger Ampel-Empfehlungen eine der wichtigsten Sofortmaßnahmen, die für eine Stress-Reduktion bei allen (Erzieher, Kinder und Eltern) sorgen würde. Diese Änderungen müssten flankiert werden von der Abschaffung des Kapitels VIII des Sozialgesetzbuchs und der Länder-Kindertagesstättengesetze, die festlegen, dass Eltern einen Anspruch auf einen Platz in einer Kinderkrippe quasi ab Geburt haben und die genau dadurch Stress-Hauptursache sind. 


\begin{tabular}{|c|c|c|c|}
\hline \multirow{2}{*}{$\begin{array}{l}\text { dgkjf } \\
\text { Kriterium }\end{array}$} & \multicolumn{3}{|c|}{ Kinderkrippen-Ampel für Rat suchende Eltern (Ausschnitt) } \\
\hline & $\begin{array}{l}\text { GRÜN } \\
\text { geht gut }\end{array}$ & $\begin{array}{c}\text { GELB } \\
\text { bedenklich }\end{array}$ & $\begin{array}{c}\text { ROT } \\
\text { Geht überhaupt nicht }\end{array}$ \\
\hline $\begin{array}{l}\text { ab welchem } \\
\text { Alter }\end{array}$ & 30 Monate & 24 Monate & $\begin{array}{l}18 \text { Monate oder } \\
\text { früher }\end{array}$ \\
\hline $\begin{array}{l}\text { Dauer } \\
\text { pro Tag }\end{array}$ & $\begin{array}{l}3 \text { - } 4 \text { Stunden } \\
\text { tägl. Krippenunter- } \\
\text { bringung }\end{array}$ & $\begin{array}{l}4 \text { - } 5 \text { Stunden } \\
\text { tägl. Krippenunter- } \\
\text { bringung }\end{array}$ & mehr als 5 Stunden \\
\hline Eingewöhnung & 6 Wochen & 4 Wochen & weniger als 4 Wochen \\
\hline $\begin{array}{l}\text { Kind-Fachkraft- } \\
\text { Relation }\end{array}$ & $\begin{array}{l}1 \text { Erzieherin } \\
2 \text { Kinder }\end{array}$ & $\begin{array}{l}1 \text { Erzieherin } \\
3 \text { Kinder }\end{array}$ & mehr als 3 Kinder \\
\hline $\begin{array}{l}\text { Personal- } \\
\text { schlüssel }\end{array}$ & $\begin{array}{l}1 \text { Erzieherin } \\
1,5 \text { Kinder }\end{array}$ & $\begin{array}{l}1 \text { Erzieherin } \\
2 \text { Kinder }\end{array}$ & mehr als 3 Kinder \\
\hline $\begin{array}{l}\text { Bezugs- } \\
\text { Erzieherin }\end{array}$ & $\begin{array}{l}\text { eine konstante } \\
\text { Bezugsperson }\end{array}$ & $\begin{array}{l}\text { zwei Bezugspersonen } \\
\text { wechseln }\end{array}$ & $\begin{array}{l}\text { mehr als zwei } \\
\text { Bezugspersonen } \\
\text { wechseln }\end{array}$ \\
\hline Gruppengröße & 8 Kinder & 9 bis 12 Kinder & $\begin{array}{l}\text { mehr als } 12 \\
\text { (wegen Lärm) }\end{array}$ \\
\hline $\begin{array}{l}\text { Betriebsferien } \\
6 \text { Wochen }\end{array}$ & $\begin{array}{l}\text { ja, Kind bleibt bei } \\
\text { den Eltern }\end{array}$ & nur 4 Wochen & keine \\
\hline $\begin{array}{l}\text { Kräfte ohne } \\
\text { Ausbildung }\end{array}$ & keine & $\begin{array}{l}\text { eine (aber nicht } \\
\text { Bezugs-Erzieherin) }\end{array}$ & $\begin{array}{l}\text { mehrere angelernte } \\
\text { Kräfte }\end{array}$ \\
\hline $\begin{array}{l}\text { Bindung } \\
\text { geht vor } \\
\text { Exploration }\end{array}$ & $\begin{array}{l}\text { ja, erst einen } \\
\text { sicheren Hafen } \\
\text { herstellen, von } \\
\text { dem aus die Welt } \\
\text { erobert wird }\end{array}$ & $\begin{array}{l}\text { Teilweise wird } \\
\text { Bindung in den } \\
\text { Vordergrund gestellt, } \\
\text { aber es wird nicht } \\
\text { eingehalten }\end{array}$ & $\begin{array}{l}\text { Bindung nicht so } \\
\text { relevant, Konzept } \\
\text { eher bildungslastig }\end{array}$ \\
\hline $\begin{array}{l}\text { Bildungs- und } \\
\text { Lernprogramm } \\
\text { für unter 3-Jähr. }\end{array}$ & $\begin{array}{l}\text { altersgemäße Neugier } \\
\text { zum Lernen nutzen - } \\
\text { keine Bildung }\end{array}$ & $\begin{array}{l}\text { wenig Bildung - } \\
\text { Neugier zum Lernen } \\
\text { nutzen }\end{array}$ & $\begin{array}{l}\text { strukturiertes } \\
\text { Lern-Angebot mit } \\
\text { Bildungsprogramm }\end{array}$ \\
\hline
\end{tabular}

Tabelle 1: Ausschnitt aus der dgkjf-Kinderkrippen-Ampel (siehe obiges Kapitel)

Bezüglich weiterer Forschung ist von größter Bedeutung, dass z. B. Elternbefragungen nach Angaben von Methodenkritikern keine brauchbaren Daten liefern. Methodisch ist es zwingend erforderlich nur noch

- Beobachtungsstudien durchzuführen wie die von Samel und Wedlich (in diesem Buch) - Cortisol-Speichelmessungen im Querschnitt und im Längsschnitt durchzuführen. Ohne diese beiden methodischen Schwerpunkte bleiben Studien widersprüchlich und lassen die wichtigsten Fragen offen. 
